\title{
Capecitabine maintenance therapy following docetaxel/capecitabine combination treatment in patients with metastatic breast cancer
}

\author{
ZEKI GOKHAN SURMELI ${ }^{1}$, UMUT VAROL ${ }^{2}$, BURCU CAKAR ${ }^{1}$, MUSTAFA DEGIRMENCI ${ }^{3}$, \\ CAGATAY ARSLAN ${ }^{4}$, GONUL DEMIR PISKIN $^{3}$, BAHA ZENGEL $^{5}$, BURCAK KARACA ${ }^{1}$, \\ ULUS ALI SANLI ${ }^{1}$ and RUCHAN USLU ${ }^{1}$
}

\footnotetext{
${ }^{1}$ Department of Internal Medicine, Division of Medical Oncology, Ege University Faculty of Medicine, Bornova;

${ }^{2}$ Medical Oncology Clinic, Izmir Katip Celebi University Ataturk Training and Research Hospital;

${ }^{3}$ Medical Oncology Clinic, Izmir Tepecik Training and Research Hospital; ${ }^{4}$ Medical Oncology Clinic, Izmir University, Medical Park Hospital; ${ }^{5}$ Medical Oncology Clinic, Bozyaka Education and Research Hospital, Izmir, Turkey
}

Received November 6, 2014; Accepted June 16, 2015

DOI: $10.3892 / 01.2015 .3546$

\begin{abstract}
The present study aimed to analyze the efficacy of maintenance therapy with single agent capecitabine for human epidermal growth factor receptor (HER2) negative metastatic breast cancer (MBC) patients following disease control with 6 cycles of docetaxel plus capecitabine chemotherapy as the first-line treatment. As an initial treatment, 6 cycles of docetaxel plus capecitabine followed by maintenance therapy with capecitabine were administered. A total of 55 patients received combination therapy and 48 patients proceeded to maintenance therapy: Of these, 32 patients (66.7\%) were postmenopausal and $37(77.1 \%)$ had estrogen and progesterone receptor positive disease. The median progression-free survival rate with maintenance therapy was 5.5 months (95\% CI, 0-11.4 months) and the median overall survival (OS) was 26.6 months (95\% CI, 21.8-30.1 months). The use of maintenance therapy improved previous responses in 4 patients $(8.3 \%$; 2 partial and 2 complete responses) and 32 patients (66.7\%) had stable disease. The median number of maintenance therapy cycles applied was 6.5 (range 1-28, total 441). The observation of side effects, including grade $3 / 4$ neutropenia, febrile neutropenia and fatigue was more common during combination therapy. The results of the present study indicate that maintenance with single agent capecitabine therapy is an effective and tolerable treatment option for HER2 negative MBC patients in which disease control with 6 cycles of docetaxel plus capecitabine chemotherapy is achieved in the first-line setting.
\end{abstract}

Correspondence to: Dr Umut Varol, Medical Oncology Clinic, Izmir Katip Celebi University Ataturk Training and Research Hospital,Basin Sitesi Street, Izmir 35360, Turkey

E-mail:varolumut@yahoo.com

Key words: breast cancer, capecitabine, docetaxel, maintenance therapy

\section{Introduction}

Breast cancer is the most common malignancy and the second most common cause of cancer mortality in women worldwide (1). Metastatic breast cancer (MBC) is an incurable disease and treatment aims to prolong survival and to improve or maintain quality of life by palliating disease-associated symptoms while minimizing the toxicity of treatment (2). Median survival of metastatic breast cancer is $\sim 2$ years, ranging from a months to years (3). Patients with hormone-insensitive disease and the majority of patients that do not respond to endocrine therapy are candidates for chemotherapy (4). Unlike in the adjuvant setting, standards of chemotherapy in metastatic disease are not well defined.

Combination chemotherapy provides higher response rates and longer time to progression (TTP), and is usually favored for patients with a high tumor burden, rapidly progressive disease or symptomatic visceral disease. Newer taxane-containing combination regimens, including docetaxel/capecitabine and paclitaxel/gemcitabine combinations, have been demonstrated to improve overall survival (OS) compared with single-agent taxanes, and these regimens are commonly used when a combination therapy is adopted $(2,3)$. However, the optimal duration of treatment to disease control with these regimens is unknown. In a previous meta-analysis, Gennari et al (4) reported that longer first-line chemotherapy duration was associated with prolonged progression-free survival (PFS) and marginally longer OS. Continuing chemotherapy until disease progression ceases is also reported to improve quality of life measures (5).

Capecitabine is an oral fluoropyrimidine and has marked activity in MBC. When used as a single agent, capecitabine provides response rates of $20-30 \%$, and median TTP of 2.8-7.1 months in the first and subsequent lines of treatment $(6,7)$. The incidence of neutropenia and alopecia are low; and more common toxicities, including hand-foot syndrome (HFS), diarrhea and stomatitis are readily managed with dose modifications $(8,9)$. Capecitabine may be a suitable option as 
a single agent for long-term use due to its tolerability, efficacy, and ease of oral application.

For patients who achieve a response with a chemotherapy doublet, clinicians often prefer to continue treatment with a less intensive regimen. In the clinical setting, continuing capecitabine or docetaxel/capecitabine combination is a common practice; however data regarding the efficacy of this approach is limited. The present study aims to evaluate the efficacy and safety of first-line therapy with docetaxel plus capecitabine followed by single-agent capecitabine in human epidermal growth factor receptor (HER2) negative MBC patients.

\section{Materials and methods}

Patient selection. Female patients aged $\geq 18$ years with histologically or cytologically proven HER 2 negative metastatic and/or locally advanced breast cancer who received no cytotoxic chemotherapy for MBC were selected for the study. In this retrospective cohort study, patients who received adjuvant chemotherapy $\geq 6$ months ago or endocrine therapy for metastatic disease were included. All the included patients had at $\geq 1$ radiologically measurable or clinically assessable lesion and an Eastern Cooperative Oncology Group performance score of $\leq 2$ (range, 0-5) (10). Eligible patients also had normal renal function and adequate hematological and hepatic function.

Study treatment. Treatment was initiated with docetaxel $75 \mathrm{mg} / \mathrm{m}^{2}$ administered as a $1 \mathrm{~h}$ infusion on the first day of every 21 -day cycle plus capecitabine $1,650 \mathrm{mg} / \mathrm{m}^{2} /$ day on days $1-14$ followed by a 7 -day rest period. Patients who achieved complete (CR) or partial (PR) responses or stable disease after 6 cycles of combination therapy, received maintenance therapy with capecitabine $2,000 \mathrm{mg} / \mathrm{m}^{2} /$ day on days $1-14$ followed by a 7-day rest period until progressive disease or intolerable toxicity. The defined protocol was administered to eligible patients in three oncology centers.

Efficacy and safety evaluations. Treatment responses were radiologically evaluated at 12-week intervals on the basis of Response Evaluation Criteria in Solid Tumors 1.1 guidelines (11). The best overall response achieved was reported separately for combination therapy and maintenance therapy. The primary efficacy endpoint was defined as the interval between the initiation of the first cycle of maintenance therapy and date of progression or death from any cause. Secondary endpoints were OS (measured from beginning of combination therapy to death from any cause) and the objective response rate (ORR). PFS duration was also calculated measured from beginning of combination therapy for all patient population. Toxicity evaluations were made prior to every treatment cycle and graded according to the Common Terminology Criteria for Adverse Events version 3 (CTCAEv3).

Statistical analyses. Descriptive data are expressed as frequency and central tendency measures. Survival durations were estimated using the Kaplan-Meier method, and the log-rank test was used to compare survival durations of patient subgroups. All P-values reported were two-sided and $\mathrm{P}<0.05$
Table I. Objective responses with combination therapy $(n=52)$.

\begin{tabular}{lrr}
\hline & $\mathrm{n}$ & $\%$ \\
\hline Partial response & 29 & 55.8 \\
Complete response & 3 & 5.8 \\
Stable disease & 16 & 30.7 \\
Progressive disease & 4 & 7.7 \\
\hline
\end{tabular}

Table II. Characteristics of patients who received maintenance treatment.

\begin{tabular}{|c|c|c|}
\hline Parameter & $\mathrm{n}$ & $\%$ \\
\hline Total & 48 & 100 \\
\hline Median age (range) & $52(28-70)$ & - \\
\hline \multicolumn{3}{|l|}{ Menopausal status } \\
\hline Premenopausal & 16 & 33.3 \\
\hline Postmenopausal & 32 & 66.7 \\
\hline \multicolumn{3}{|l|}{ Hormone receptor } \\
\hline Positive & 37 & 77.1 \\
\hline Negative & 11 & 22.9 \\
\hline \multicolumn{3}{|l|}{ Metastatic site } \\
\hline Visceral & 25 & 52.1 \\
\hline Non-visceral & 23 & 47.9 \\
\hline \multicolumn{3}{|l|}{ No. of tumor sites } \\
\hline 1 & 20 & 41.7 \\
\hline 2 & 19 & 39.6 \\
\hline$\geq 3$ & 9 & 18.8 \\
\hline \multicolumn{3}{|c|}{ Previous endocrine therapy } \\
\hline Adjuvant & 18 & 37.5 \\
\hline Metastatic & 17 & 35.4 \\
\hline Adjuvant chemotherapy & 28 & 58.3 \\
\hline Anthracycline exposure & 28 & 58.3 \\
\hline Taxane exposure & 24 & 50.0 \\
\hline
\end{tabular}

was considered to indicate a statistically significant difference. Statistical analyses were performed using the SPSS software, version 17 (SPSS, Inc., Chicago, IL, USA.

\section{Results}

Patient characteristics. Between June 2009 and June 2012, 55 patients were enrolled in the study and received docetaxel plus capecitabine regimen. During combination chemotherapy one patient died, one patient was lost to follow-up and one patient developed intolerable hand-foot syndrome. Response assessments were made for 52 patients. After 6 cycles of combination chemotherapy 29 patients had PR, three patients had CR and 16 patients had stable disease. The ORR for combination chemotherapy was $61.6 \%$ (Table I). A total of 4 patients had progressive disease, one patient died, one patient was lost to follow-up, and one patient had intolerable HFS and did not receive maintenance therapy. Forty-eight patients 

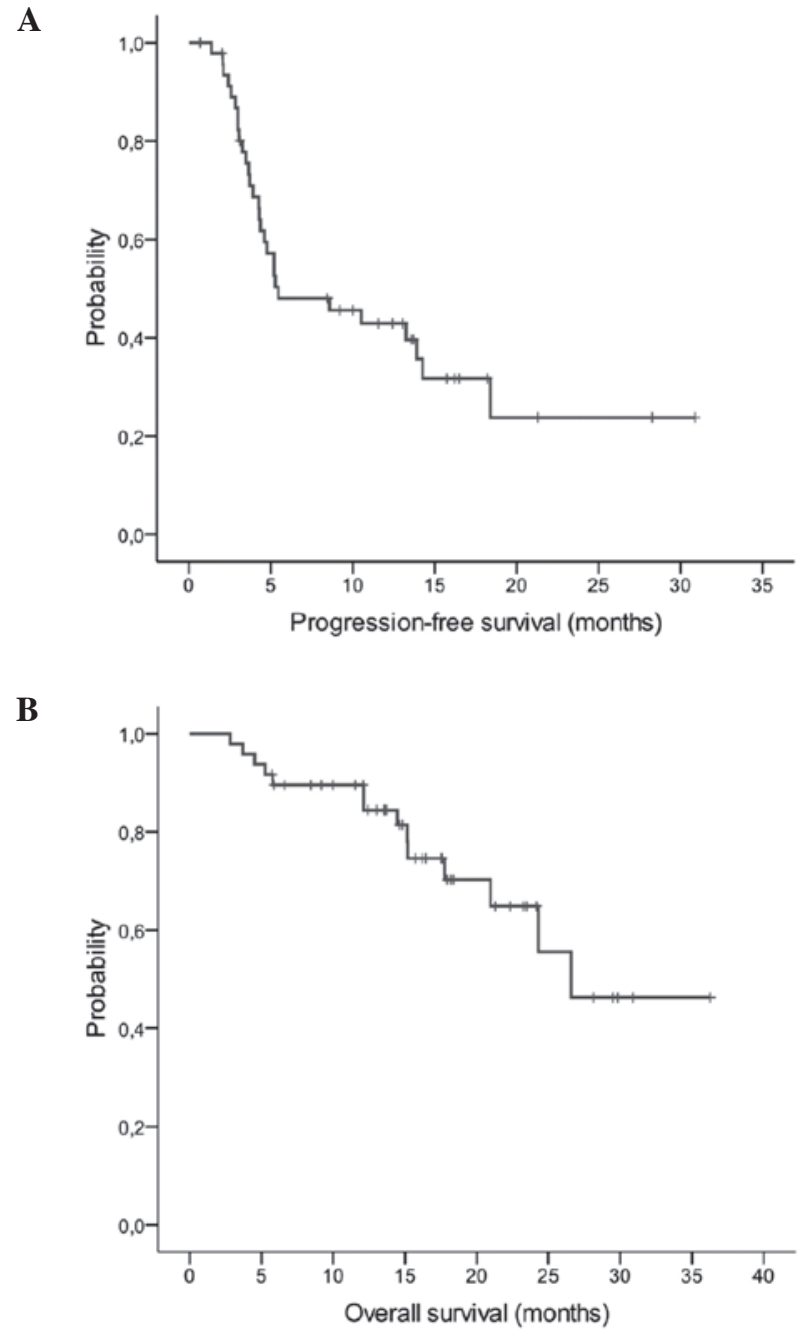

Figure 1. (A) Progression-free survival curve (number of events=29) and (B) overall survival curve (number of events=14).

proceeded to maintenance phase and received $\geq 1$ cycle of single-agent capecitabine. The median age of the patient group was 52 years (range 28-70 years). A total of 32 patients (66.7\%) were postmenopausal and $37(77.1 \%)$ had estrogen and progesterone receptor (hormone receptor) positive disease. The number of metastatic sites was one site in 20 patients, two sites in 19 patients and three or more sites in 9 patients. The most common metastatic sites were bone (75\%), lymph nodes (33.3\%), lungs (27.1\%) and liver (14.6\%). Twenty-five patients (52.1\%) had at least one visceral metastatic site, and metastases were limited to bone, lymph nodes or soft tissue in 23 patients (47.9\%). Seventeen patients (35.4\%) received previous endocrine therapy for MBC and 29 patients (60.4\%) had received endocrine therapy in adjuvant and/or metastatic setting. Adjuvant chemotherapy was administered to 28 patients (58.3\%). All adjuvant regimens included an anthracycline, and 24 patients $(50 \%)$ received a taxane in the adjuvant setting. The baseline characteristics of the patient population are presented in Table II.

Efficacy. The median follow-up duration following the initiation of maintenance capecitabine treatment was 17.6 months. The median number of maintenance capecitabine cycles
Table III. PFS durations in patient subgroups.

\begin{tabular}{lrrr}
\hline Subgroup & $\begin{array}{c}\text { Median PFS } \\
\text { (months) }\end{array}$ & 95\% CI & P-value \\
\hline $\begin{array}{l}\text { Menopausal status } \\
\text { Pre- (n=16) }\end{array}$ & 10.5 & $0.9-20.1$ & 0.923 \\
$\quad$ Post- (n=32) & 5.4 & $0-10.9$ & \\
Hormone receptor & & & \\
$\quad$ Positive (n=37) & 13.2 & $6.1-20.4$ & $\mathbf{< 0 . 0 0 1}$ \\
$\quad$ Negative (n=11) & 3.7 & $2.5-4.80$ & \\
No. of metastatic sites & & & \\
1 (n=20) & 13.2 & $3-23.5$ & 0.230 \\
$\geq 2$ (n=28) & 4.8 & $3.3-6.20$ & \\
Metastatic site & & & \\
$\quad$ Non-visceral (n=23) & 14.3 & $8-20.5$ & $\mathbf{0 . 0 1 6}$ \\
Visceral (n=25) & 4.3 & $3.3-5.30$ & \\
Previous taxane & & & \\
exposure & & & \\
No (n=24) & 10.5 & $0-22$ & 0.065 \\
Yes (n=24) & 4.4 & $3.3-5.40$ & \\
Previous anthracycline & & & \\
exposure & & & \\
$\quad$ No (n=20) & 5.8 & $0-13.8$ & 0.271 \\
Yes (n=28) & 5.2 & $0-10.7$ & \\
\hline CI, confidence interval; PFS, progression-free survival. & \\
\hline
\end{tabular}

was 6.5 (range 1-28, total 441). The median PFS with single agent capecitabine was 5.5 months $(95 \%$ CI, 0-11.4) with 29 events (Fig. 1A). In the maintenance phase, 4 patients (8.3\%) had improved responses with single-agent capecitabine; 2 patients prior PR further improved and 2 patients improved from PR to CR. Thirty-two patients (66.7\%) had stable disease. Twenty-one patients received maintenance therapy for $\geq 6$ months, 15 patients for $\geq 12$ months and 5 patients for $\geq 18$ months. At the time of analyses 5 patients were still receiving maintenance therapy. Hormone receptor positive patients (13.2 vs. 3.7 months, $\mathrm{P}<0.001)$ and patients with no visceral metastases ( 14.3 vs. 4.3 months, $P<0.016$ ) had significantly longer PFS durations (Table III). Fourteen patients died and the median OS after the initiation of maintenance therapy was 26.6 months (95\% CI, 21.8-30.1; Fig. 1B). The treatment regimen including the combination phase provided a median PFS of 9.8 months (95\% CI, 8.4-11.1) for all patient groups $(\mathrm{n}=55)$.

Safety. Adverse events were assessed in 36 patients. The most common toxicities $(>10 \%)$ were HFS, neutropenia and fatigue with combination therapy and HFS with maintenance therapy. Febrile neutropenia, grade $3 / 4$ neutropenia and grade $1 / 2$ fatigue were more common during combination therapy (Table IV). Capecitabine was discontinued in 5 patients due to intolerable toxicity during maintenance therapy ( $3 \mathrm{HFS}, 1$ edema and 1 abnormal vision). During combination therapy dose reductions of capecitabine were performed in 9 patients (16.4\%) and 
Table IV. Treatment-associated adverse events.

\begin{tabular}{|c|c|c|c|c|}
\hline \multirow[b]{2}{*}{ Event } & \multicolumn{2}{|c|}{ Docetaxel/capecitabine } & \multicolumn{2}{|c|}{ Capecitabine maintenance } \\
\hline & Grade $1 / 2(\%)$ & Grade 3/4 (\%) & Grade $1 / 2(\%)$ & Grade $3 / 4(\%)$ \\
\hline Neutropenia & $2(5.6)$ & $5(13.9)$ & $2(5.6)$ & $1(2.8)$ \\
\hline Febrile neutropenia & 0 & $3(8.3)$ & 0 & 0 \\
\hline Hand foot syndrome & $8(22.2)$ & $9(25)$ & $7(19.4)$ & $5(13.9)$ \\
\hline Fatigue & $8(22.2)$ & $1(2.8)$ & $3(8.3)$ & 0 \\
\hline Stomatitis & $3(8.3)$ & $1(2.8)$ & $2(5.6)$ & 0 \\
\hline
\end{tabular}

reduction of docetaxel were performed in 11 patients $(20 \%)$. Seven patients $(14.6 \%)$ required a dose reduction during maintenance therapy. No treatment-associated mortality occurred.

\section{Discussion}

The results of the present study indicate that maintenance therapy with single agent capecitabine following first-line treatment with 6 cycles of docetaxel plus capecitabine therapy is an effective treatment option for treatment of patients with HER 2 negative MBC. The maintenance therapy provided a median PFS duration of 5.5 months and treatment regimens that included combination therapy provided a median PFS duration of 9.8 months. Efficacy outcomes of this regimen are in the range of data reported for docetaxel and capecitabine combination. In the first-line setting this combination is reported to provide a PFS duration of 8.5-10 months and an ORR of $39-74 \%(12,13)$. Although a control arm was not included in the present study, the TTP following 6 cycles of taxane with combination therapy without maintenance was 3.8 months in a previous study (14). Objective responses were preserved or improved in $75 \%$ of patients. Toxicity profiles were compatible with a previous study (3). Capecitabine maintenance resulted in reduced neutropenia, febrile neutropenia and fatigue compared with combination treatment. During maintenance therapy incidence of grade $3 / 4$ neutropenia was only $2.8 \%$ and no febrile neutropenia was observed. The main toxicity event associated with capecitabine therapy was HFS; $13.9 \%$ of patients developed grade $3 / 4$ HFS during maintenance therapy.

To the best of our knowledge, the efficacy of capecitabine maintenance therapy has only been evaluated in one previous study (15). Patients were administered single agent capecitabine after a positive response to capecitabine plus docetaxel or vinorelbine in the first or second-line setting and the median TTP with maintenance was 4.4 months. In this study $81.4 \%$ of the patient group maintained the response to combination regimen by maintenance, $1.7 \%$ demonstrated an improvement from $\mathrm{PR}$ to $\mathrm{CR}$ in maintenance setting.

In management of $\mathrm{MBC}$, newer combination regimens may offer a survival rate advantage, however increased toxicity may limit tolerability. When disease control is achieved, possible options include, discontinuing treatment until progression or continuing with the same or a less intensive regimen including hormonal therapy. For hormone receptor-positive patients, maintenance with endocrine therapy, such as tamoxifen, anastrazole, letrozole or exemestane, is preferred by a number of clinicians. Although available data is limited, a study by Bertelli et al (16), supports this approach. Letrozole provided a median TTP of 18.5 months in 58 postmenopausal patients who attained disease control with first-line chemotherapy. The benefit of maintenance therapy with modern chemotherapy regimens is still a matter of debate. Three trials with different designs assessed the efficacy of maintenance chemotherapy following taxane-containing combination chemotherapy in HER2 negative patients. In MANTA1 study, maintenance with paclitaxel after anthracycline-paclitaxel combination provided no benefit in terms of PFS and OS compared with control group (17). However, it should be noted that around $60 \%$ of the patients had received concurrent endocrine therapy in both arms. In GEICAM 2001-01 study, maintenance with pegylated liposomal doxorubicin significantly prolonged TTP (8.4 vs. 5.1 months), but not OS compared with observation and patients were not allowed to receive hormonal therapy in both arms (18). In a recent study by the Korean Cancer Study Group (KCSG), patients received 6 cycles of paclitaxel plus gemcitabine and those who achieved disease control were randomized to receive maintenance with the same regimen until progression or observation (14); again, endocrine therapy was not allowed. In this study, patients in the maintenance arm received additional 6 cycles (median) of therapy, and the results demonstrated a superior OS (32.3 vs. 23.5 months) and PFS with maintenance therapy; however the incidence of grade $3 / 4$ neutropenia was also increased $(61 \%)$.

In conclusion, present data about the benefit of maintenance therapy is inconclusive, and decision of maintenance therapy should be based on the disease characteristics and patient preferences (19). The results of the present study demonstrate that single-agent capecitabine after docetaxel and capecitabine combination is safe, efficient and feasible, and may be considered as an option when maintenance therapy is preferred.

\section{References}

1. McPherson K, Steel CM, and Dixon JM: Breast cancer-epidemiology, risk factors, and genetics. BMJ 321: 624-628, 2000.

2. Albain KS, Nag SM, Calderillo-Ruiz G, Jordaan JP, Llombart AC, Pluzanska A, Rolski J, Melemed AS, Reyes-Vidal JM, Sekhon JS, et al: Gemcitabine plus Paclitaxel versus Paclitaxel monotherapy in patients with metastatic breast cancer and prior anthracycline treatment. J Clin Oncol 26: 3950-3957, 2008.

3. O'Shaughnessy J, Miles D, Vukelja S, Moiseyenko V, Ayoub JP, Cervantes G, Fumoleau P, Jones S, Lui WY, Mauriac L, et al: Superior survival with capecitabine plus docetaxel combination therapy in anthracycline-pretreated patients with advanced breast cancer: Phase III trial results. J Clin Oncol 20: 2812-2823, 2002. 
4. Gennari A, Stockler M, Puntoni M, Sormani M, Nanni O, Amadori D, Wilcken N, D'Amico M, DeCensi A and Bruzzi P: Duration of chemotherapy for metastatic breast cancer: A systematic review and meta-analysis of randomized clinical trials. J Clin Oncol29: 2144-2149, 2011.

5. Coates A, Gebski V, Bishop JF, Jeal PN, Woods RL, Snyder R, Tattersall MH, Byrne M, Harvey V, Gill G, et al: Improving the quality of life during chemotherapy for advanced breast cancer. A comparison of intermittent and continuous treatment strategies. N Engl J Med 317: 1490-1495, 1987.

6. O'Shaughnessy JA, Kaufmann M, Siedentopf F, Dalivoust P, Debled M, Robert NJ and Harbeck N: Capecitabine monotherapy: Review of studies in first-line HER-2-negative metastatic breast cancer. Oncologist 17: 476-484, 2012.

7. Blum JL, Barrios CH, Feldman N, Verma S, McKenna EF, Lee LF, Scotto N and Gralow J: Pooled analysis of individual patient data from capecitabine monotherapy clinical trials in locally advanced or metastatic breast cancer. Breast Cancer Res Treat 136: 777-788, 2012.

8. Rossi D, Alessandroni P, Catalano V, Giordani P, Fedeli SL, Fedeli A, Baldelli AM, Casadei V, Ceccolini M and Catalano G: Safety profile and activity of lower capecitabine dose in patients with metastatic breast cancer. Clin Breast Cancer7: 857-860, 2007.

9. Hennessy BT, Gauthier AM, Michaud LB, Hortobagyi G and Valero V: Lower dose capecitabine has a more favorable therapeutic index in metastatic breast cancer: Retrospective analysis of patients treated at M. D. Anderson Cancer Center and a review of capecitabine toxicity in the literature. Ann Oncol 16 : 1289-1296, 2005.

10. Oken MM, Creech RH, Tormey DC, Horton J, Davis TE, McFadden ET and Carbone PP: Toxicity and response criteria of the Eastern Cooperative Oncology Group. Am J Clin Oncol 5 : 649-655, 1982

11. Eisenhauer EA, Therasse P, Bogaerts J, Schwartz LH, Sargent D, Ford R, Dancey J, Arbuck S, Gwyther S, Mooney M, et al: New response evaluation criteria in solid tumours: Revised RECIST guideline (version 1.1). Eur J Cancer 45: 228-247, 2009.

12. Vici P, Giotta F, Di Lauro L, Sergi D, Vizza E, Mariani L, Latorre A, Pizzuti L, D'Amico C, Giannarelli D, et al: A multicenter phase II randomized trial of docetaxel/gemcitabine versus docetaxel/capecitabine as first-line treatment for advanced breast cancer: A Gruppo Oncologico Italia Meridionale study. Oncology 81: 230-236, 2011.
13. Soto C, Torrecillas L, Reyes S, Ramirez M, Perez L, Cervantes G, Gonzalez F, Tellez E, Cortes P and Benitez H: Capecitabine (X) and taxanes in patients (pts) with anthracycline-pretreated metastatic breast cancer (MBC): Sequential vs. combined therapy results from a MOSG randomized phase III trial. J Clin Oncol (Meeting abstracts) 24: 570, 2006.

14. Park YH, Jung KH, Im SA, Sohn JH, Ro J, Ahn JH, Kim SB, Nam BH, Oh Y, Han SW, et al: Phase III, multicenter, randomized trial of maintenance chemotherapy versus observation in patients with metastatic breast cancer after achieving disease control with six cycles of gemcitabine plus paclitaxel as first-line chemotherapy: KCSG-BR07-02. J Clin Oncol 31: 1732-1739, 2013

15. Huang H, Jiang Z, Wang T, Zhang S, Bian L, Cao Y, Wu S and Song S: Single-agent capecitabine maintenance therapy after response to capecitabine-based combination chemotherapy in patients with metastatic breast cancer. Anticancer Drugs 23: 718-723, 2012.

16. Bertelli G, Garrone O, Bertolotti L, Occelli M, Conforti S, Marzano N, Febbraro A, Carlini P, Liossi C, Del Mastro L, et al: Maintenance hormone therapy with letrozole after first-line chemotherapy for advanced breast cancer. Oncology 68: 364-370, 2005.

17. Gennari A, Amadori D, De Lena M, Nanni O, Bruzzi P, Lorusso V, Manzione L and Conte PF: Lack of benefit of maintenance paclitaxel in first-line chemotherapy in metastatic breast cancer. J Clin Oncol 24: 3912-3918, 2006.

18. Alba E, Ruiz-Borrego M, Margelí M, Rodríguez-Lescure A, Sánchez-Rovira P, Ruiz A, Mel-Lorenzo JR, Ramos-Vázquez M, Ribelles N,Calvo E, et al: Maintenance treatment with pegylated liposomal doxorubicin versus observation following induction chemotherapy for metastatic breast cancer: GEICAM 2001-01 study. Breast Cancer Res Treat 122: 169-176, 2010.

19. Carrick S, Parker S, Thornton CE, Ghersi D, Simes J and Wilcken N: Single agent versus combination chemotherapy for metastatic breast cancer. Cochrane Database Syst Rev: Apr 15, 2009 (Epub ahead of print). 